\title{
Chromatophorenanordnungen in emersen Thalli von Fucus vesiculosus unter verschiedenen Lichtbedingungen
}

\author{
W. Nultsch, U. Rüffer \& J. Pfau \\ Botanisches Institut der Universität Marburg; \\ Labnberge, D-3550 Marburg, Bundesrepublik Deutschland
}

\begin{abstract}
Chromatophore arrangements in exposed thalli of Fucus vesiculosus under different light conditions. The effects of light and darkness on chromatophore arrangements in Fucus vesiculosus thalli exposed to air with one side (half dry) or with both sides (dry) were investigated. Low-intensity arrangement, high-intensity arrangement and dark arrangement were induced in submerged thalli. Then the thalli were exposed to air while light conditions were either kept constant or were changed. Independently of the light conditions, in all thalli exposed to air the chromatophores arranged more or less in the inner area of the cells, in epidermal cells as well as in cortical cells. Thus the chromatophore arrangements observed in submerged thalli differ in some respect from those exposed to low-tide conditions. Based on these observations the physiological and ecological role of chromatophore displacements in seaweeds is discussed.
\end{abstract}

\section{EINLEITUNG}

In einer vorausgegangenen Arbeit (Rüffer et al., 1977) wurden die bei Fucus vesiculosis nach Bestrahlung mit Starklicht bzw. Schwachlicht sowie nach Verdunkelung auftretenden charakteristischen Chromatophorenanordnungen in den verschiedenen Zellschichten des Gewebethallus genau beschrieben. Hierbei fanden Thalli Verwendung, die sich während der gesamten Dauer der Bestrahlung in Durchflußküvetten, also im submersen Zustand befanden. Dies entspricht insofern nicht ganz den natürlichen Verhältnissen, als $F$. vesiculosus, eine typische Gezeitenalge des Helgoländer Felswatts, bei Niedrigwasser regelmäßig für eine längere oder kürzere Zeit trockenfällt und gerade während dieser Zeit der direkten Sonnenbestrahlung ausgesetzt ist. Häufig liegen die Thalli einer felsigen Unterlage oder anderen Algen auf, so daß nur die eine Seite der Luft exponiert ist. Solche Thalli werden im folgenden als "halbtrocken" bezeichnet. Nicht selten hängen sie jedoch von größeren Blöcken, an denen sie festgewachsen sind, so herab, daß beide Seiten des flächigen Thallus der Luft ausgesetzt sind. Solche Thalli können nach längerer Zeit so stark austrocknen, daß sie eine fast papierne Konsistenz annehmen. Beidseitig der Luft exponierte Thalli werden im folgenden als "trocken" bezeichnet.

Schon die klassischen Untersuchungen von Montfort (1937) und Biebl (1938) haben ergeben, daß $F$. vesiculosus eine erhebliche Trockenresistenz besitzt. Dies wurde durch 
neuere Untersuchungen von Schramm (1968) und Ried (1969) bestätigt. Darüber hinaus haben die Arbeiten von Stocker \& Holdheide (1938) und Kremer \& Schmitz (1973) gezeigt, daß Fucus-Thalli auch im emersen Zustand zu beträchtlichen Photosyntheseleistungen befähigt sind, also die Phase des Trockenfallens nicht nur passiv ïberstehen. Es war daher von Interesse, den Einfluß des Lichtes auf die Chromatophorenanordnung im emersen Zustand zu untersuchen.

\section{MATERIAL UND METHODIK}

Thalli von Fucus vesiculosus wurden an geeigneten Standorten des Helgoländer Felswatts gesammelt und entweder an Ort und Stelle fixiert oder in thermokonstanten Räumen den gewünschten Versuchsbedingungen ausgesetzt und dann fixiert. Bezüglich der Bestrahlungsversuche im Labor sei auf Rüffer et al. (1977) verwiesen. Das für die Messung der Transmissionsänderungen benutzte Mikrophotometer ist bei Pfau et al. (1974) beschrieben. Im Regelfall wurden die Thallusteile vor Versuchsbeginn entweder mit Schwachlicht (1000 Lux) einen Tag oder mit Starklicht 4 Stunden lang in Seewasser vorbelichtet oder 2 Tage lang vorverdunkelt und dann mit Zellstoff oberflächlich abgetrocknet. Um die "halbtrockene" Exposition zu simulieren, wurden sie auf mehrere Lagen mit Seewasser getränkten Zellstoffs gelegt und so auf der einen Seite feucht gehalten. Bestrahlt und untersucht wurde die der Luft exponierte Seite. Zur Simulierung der "trockenen" Exposition wurden die Thallusstücke zwischen Plastikblöcken eingespannt. Da nach Schramm (1968) ein Absinken des Wassergehaltes unter $30 \%$ (bezogen auf den Sättigungswassergehalt) rasch zu irreversiblen Schäden führt und ein zu starker und schneller Wasserentzug ohnehin Plastidenverlagerungen unmöglich macht, wurde in allen Fällen ein durch Seewasser geleiteter Luftstrom (Waschflasche) über die Thalli geführt. Die Trocknungsversuche in Starklicht, Schwachlicht oder Dunkelheit erstreckten sich über 2-24 Stunden.

Nach Versuchsende wurden die Thallusstücke sogleich in einer modifizierten Nawaschin-Lösung fixiert, in Seewasser abnehmender Salzkonzentration ausgewaschen, in essigsaurer Säurefuchsinlösung gefärbt und mit der Hand geschnitten. Wegen weiterer methodischer Einzelheiten sei auf Rüffer et al. (1977) verwiesen.

\section{ERGEBNISSE}

Alle Versuche, die lichtinduzierten Chromatophorenverlagerungen bei "halbtrockenen" und "trockenen" Thalli durch mikrophotometrische Messung der Transmissionsänderungen zu erfassen, verliefen erfolglos, da die Thalli während des Trocknungsvorganges stark schrumpfen. Dabei nimmt die Thallusbreite um etwa die Hälfte, die Länge um etwa $1 / 3$ ab. Dies ließ sich durch mikroskopische Untersuchungen bestätigen. In Querschnitten durch trockene Thalli, die in $\mathrm{Ol}$ untersucht wurden, ist bei gleicher Vergrößerung die Anzahl der Epidermiszellen etwa doppelt so groß wie in einem entsprechenden Abschnitt submers gehaltener Thalli (vgl. Abb. 1 A, B). Die Schrumpfung führt im gemessenen Feld also zu einer beträchtlichen Erhöhung der Chromatophorenzahl, im Maximum auf das 

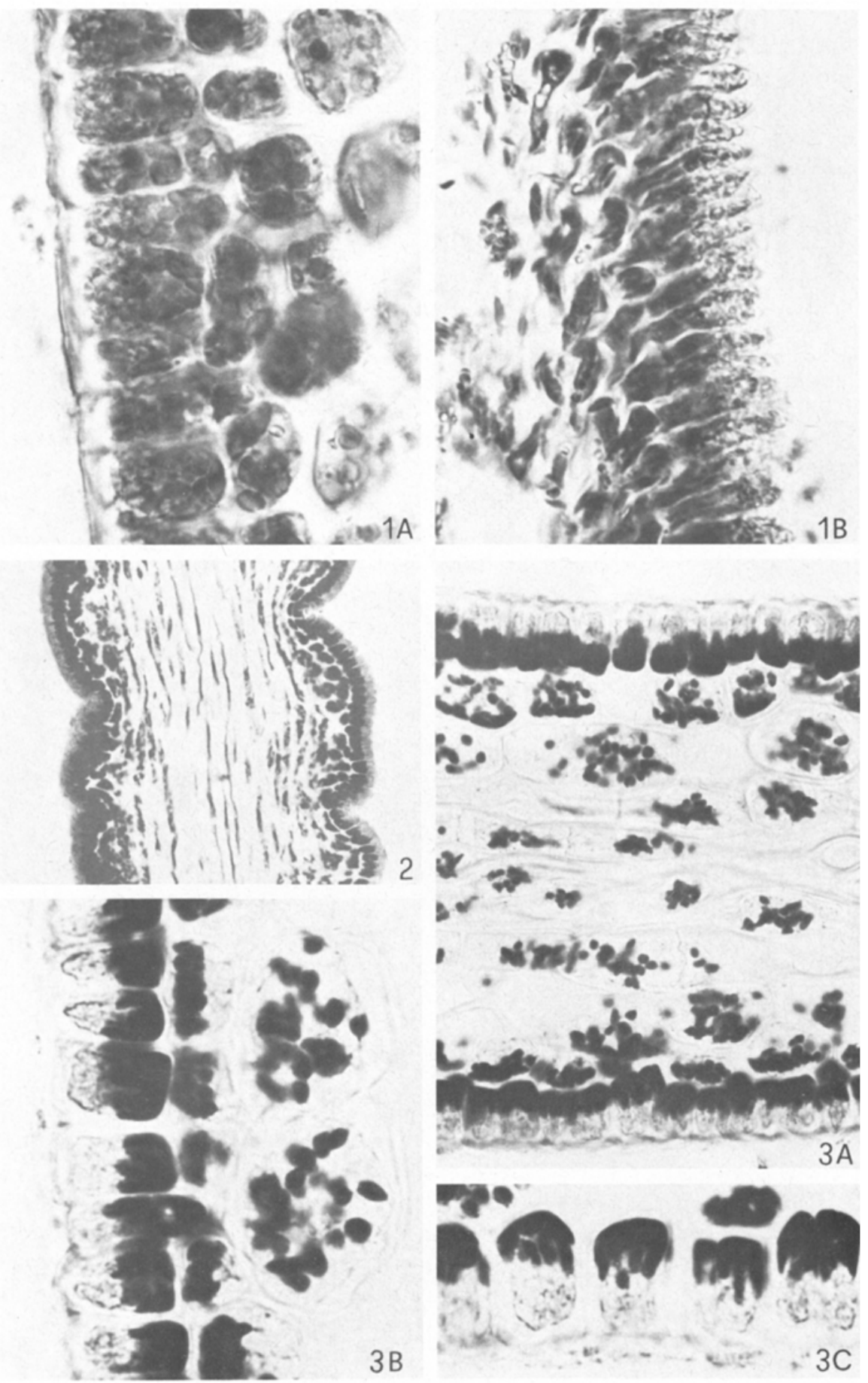
Dreifache. Infolgedessen nehmen die Transmissionswerte während des Trocknungsvorganges $a b$, und $z$ war so stark, daß die durch lichtinduzierte Chromatophorenverlagerungen hervorgerufenen Transmissionsänderungen hierdurch überdeckt werden und infolgedessen nicht mehr meßbar sind. Übrigens wird beim Trocknen auch die Dicke der Thalli auf etwa $1 / 3$ reduziert, was jedoch für die Transmissionsmessungen von untergeordneter Bedeutung sein dürfte.

Wie ein Vergleich von Querschnitten durch einen submersen und einen stark getrockneten Thallus zeigt, verliert vor allem die Zwischensubstanz Wasser. Dies äußert sich gleichermaßen in einer Zunahme der Lichtbrechung der Matrix wie auch in einem stärkeren Zusammenrücken der Zellen, und zwar besonders im Bereich der zentralen Hyphen (Abb. 2). Die Epidermiszellen werden, im Querschnitt betrachtet, vor allem schmaler (Abb. $1 \mathrm{~B}$ ). Die Rindenzellen vermindern ihr Volumen entsprechend. Die Plastiden kugeln sich durch den Wasserentzug nicht ab, sondern schrumpfen, wobei sie die verschiedensten Gestalten annehmen.

Uberführt man einen papiertrockenen Thallus in Seewasser, so macht er makroskopisch nach 15 min wieder einen "normalen" Eindruck. Die Wasseraufnahme erfolgt also, zumindest durch die Matrix, sehr schnell. Thalli, die in feuchter Atmosphäre frei auf einer nassen Unterlage liegen (halbtrockene Thalli), bleiben turgeszent.

\section{Luftexposition unter Schwachlichtbestrahlung}

Werden Thalli, die mit Schwachlicht (1000 Lux) submers vorbelichtet waren, unter Beibehaltung der Bestrahlungsintensität der Luft exponiert, so weicht mit zunehmender Expositionsdauer die Anordnung der Chromatophoren in den Zellen von der typischen Schwachlichtstellung (Rüffer et al., 1977) ab. Dies ist in geringerem Maße auch bei den "halbtrockenen" Thalli der Fall. In der Epidermis wandert ein Teil der Plastiden aus dem plasmatischen Wandbelag in das Zellinnere (Abb. 3 B, C). Ähnliche Verlagerungen sind in den Rindenzellen zu beobachten. Hier liegen die Plastiden meist unregelmäßig verteilt sowohl an der Oberfläche wie auch im Innenraum der Zelle (Abb. 3 A, B, 4 A). In den Zellen der inneren Rinde und in den Hyphen scharen sich die Chromatophoren häufig locker um den Kern (Abb. 3 A, 4 C). In den äußeren Rindenzellen ist dies seltener der Fall (Abb. 4 B). Bei diesen findet manchmal nur eine Verlagerung zur Thallusaußenseite statt (Abb. 3 B). Letztere wie auch die unregelmäßige Verteilung der Chromatophoren im Plasmawandbelag und im Zellinnenraum ähnelt gewissen bei submersen Thalli zu beobachtenden Ubergangspositionen von der Schwachlicht- zur Starklichtstellung.

Waren die Thalli vorher in Seewasser 4 Std lang mit $20000 \mathrm{Lux}$ bestrahlt worden, so ändert sich die Plastidenanordnung während des Trocknens bei gleichzeitiger Bestrahlung mit 1000 Lux kaum.

Abb. 1: Querschnitte durch Epidermis und Rinde lebender Thalli. (A) submerser Thallus, in Seewas$\operatorname{ser}(1000: 1)$; (B) stark getrockneter Thallus, in $\mathrm{Ol}(1000: 1)$

Abb. 2: Querschnitt durch emen stark getrockneten Thallus, fixiert und gefärbt $(200: 1)$

Abb. 3: Querschnitte durch Thalli, die nach submerser Schwachlichtvorbestrahlung 4 Std. bei 1000 Lux getrocknet wurden. (A) in beiden Rindenschichten sind die Plastiden zur Lichtseite (unten) gewandert (500:1); (B) Epidermis und Rinde der lichtzugewandten Seite (1000:1); (C) Epidermis $(1000: 1)$ 


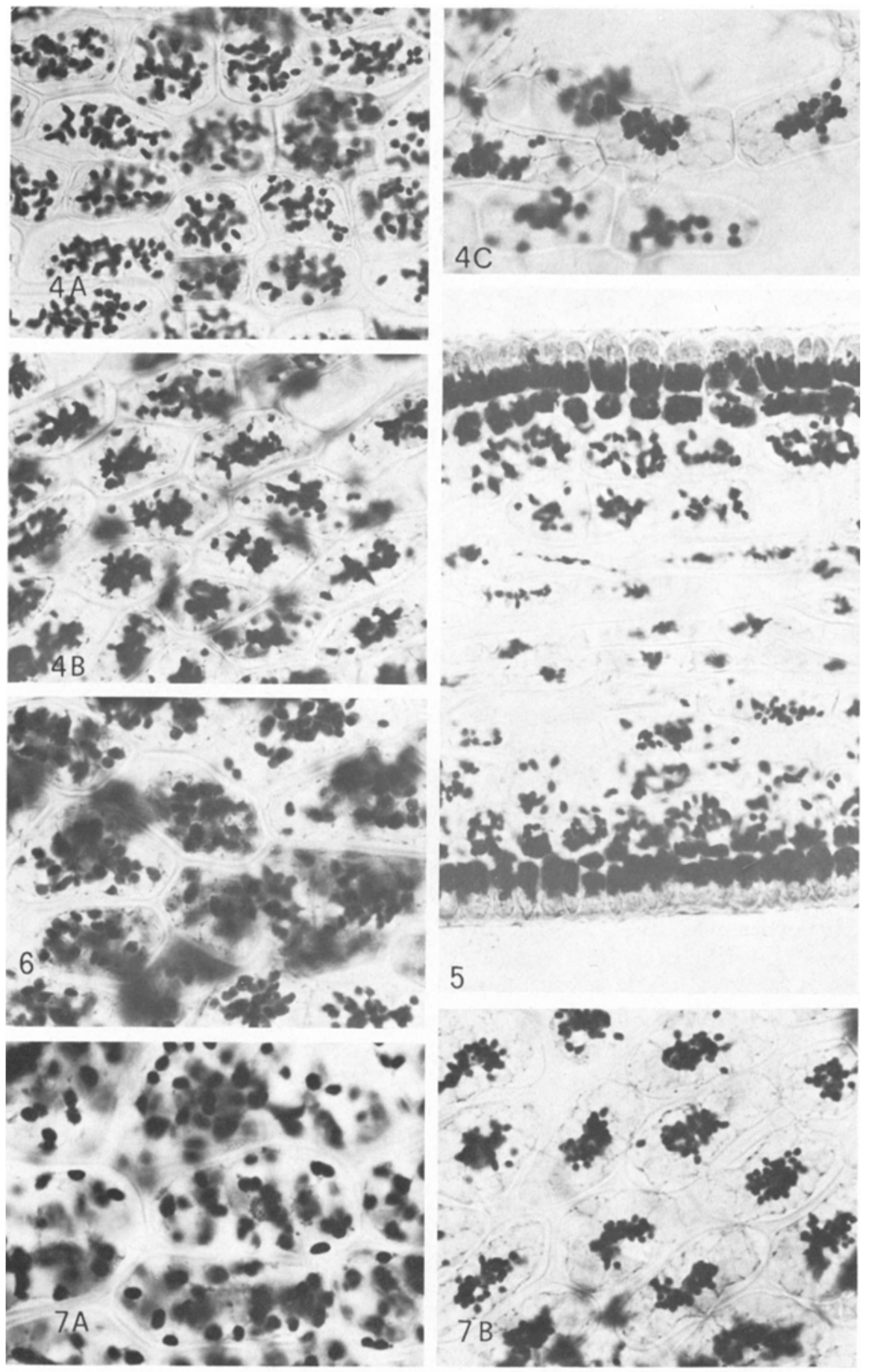


Eine länger dauernde (bis zu $24 \mathrm{Std}$ ), aber nicht vollständige Trocknung vertrugen die Fucus-Thalli gut. Nach Ubertragung in Seewasser unter Beibehaltung der Schwachlichtbestrahlung machten die Zellen schon nach 4 Std wieder einen normalen, voll vitalen Eindruck. Allerdings wird die typische Schwachlichtlage nur langsam wieder erreicht, z. B. nach vierstündiger Trocknung erst in 1 bis 2 Tagen. Werden die Thalli anschließend in Seewasser einer Starklichtbestrahlung ausgesetzt, so zeigen sie nach relativ kurzer Zeit eine deutliche Starklichtstellung mit starken zentralen Aggregationen.

\section{Luftexposition unter Starklichtbestrahlung}

Thalli, die mit Schwachlicht (1000 Lux) vorbelichtet waren und dann der Luft bei Starklichtbestrahlung (20000 Lux) exponiert wurden, zeigten keine grundsätzlich andere Chromatophorenanordnung als die submersen Thalli (vgl. Rüffer et al., 1977). Allerdings trat bei den "halbtrockenen" Thalli die ausgeprägte Starklichtstellung, insbesondere die zentrale Zusammenballung um den Kern, eher auf als bei den "trockenen". Bei den letzteren waren die Plastiden meist im Plasmawandbelag und im Zellinnenraum verteilt (Abb. 5, 6), wobei die Lichtseite bevorzugt wurde, also ähnlich wie in den Schwachlichtversuchen. Ebenso wie dort wichen die Epidermisplastiden, im Gegensatz zu den submersen Thalli, nicht oder kaum zur Basis zurück.

Bei Thalli, die vorher 4 Std lang submers mit Starklicht (20 000 Lux) bestrahlt worden waren, änderte sich die Plastidenanordnung während der Luftexposition unter Beibehaltung der Starklichtbestrahlung nicht.

\section{Luftexposition unter Dunkelhaltung}

Wurden die Thalli in Seewasser vorverdunkelt und dann im Dunkeln getrocknet, so behielten die Chromatophoren der "halbtrockenen" Thalli auch nach Tagen eine reine Dunkelstellung bei. Dagegen war bei "trockenen" Stücken bisweilen schon nach 4 Std, meist aber erst nach einer längeren Zeit eine teilweise Verlagerung der Plastiden in den Zellinnenraum (Abb. 8, 9) und eine unregelmäßige, lockere Verteilung ohne regelrechte Aggregationen zu beobachten, wie sie auch für die im Schwachlicht gehaltenen Thalli beschrieben wurde. In Seewasser überführt nahmen die Chromatophoren nach etwa einem Tag wieder die normale Dunkellage an. Ähnlich verhielten sich die Chromatophoren von

Abb. 4: Flächenschnitte durch Thalli, die nach submerser Schwachlichtvorbestrahlung bei 1000 Lux getrocknet wurden. (A) Rinde, 4 Std. getrocknet (400:1); (B) Rinde, 16 Std. getrocknet (500:1); (C) Mark, $31 / 2$ Std. getrocknet $(500: 1)$

Abb. 5: Querschnitt durch einen nach submerser Schwachlichtvorbestrahlung 16 Std. bei 20000 Lux getrockneten Thallus $(400: 1)$

Abb. 6: Flächenschnitt durch die Rinde eines wie in Abb. 5 behandelten Thallus (500:1)

Abb. 7: Flächenschnitte durch die Rinde von Thalli, die nach Schwachlichtvorbestrahlung in normalem Seewasser 20 Std. lang in hypertonischem Seewasser mit 1000 Lux bestrahlt wurden. (A) 4 fach konzentriertes Seewasser (700:1); (B) 2fach konzentriertes Seewasser (400:1) 

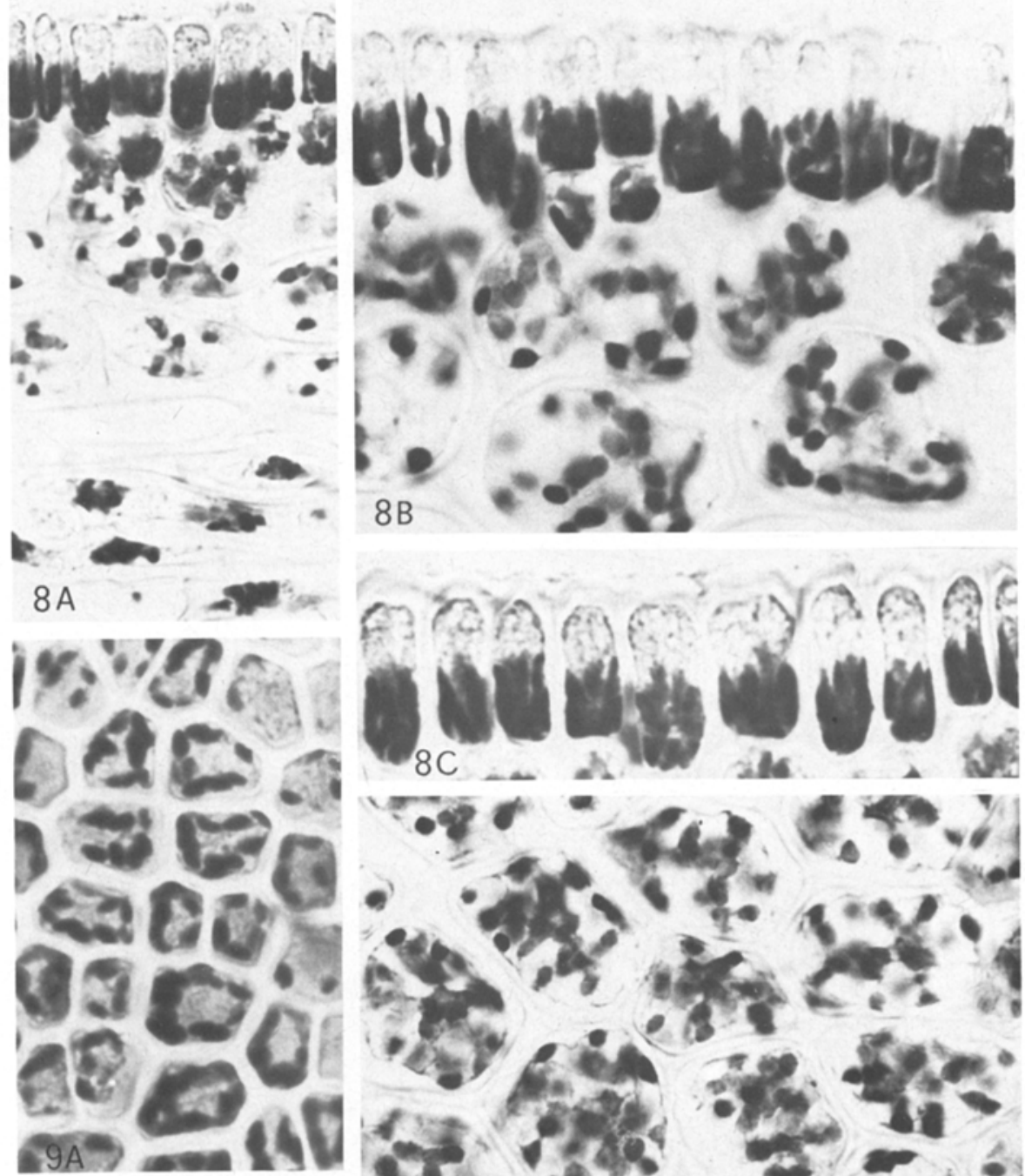

$8 \mathrm{~A} \cdot \mathrm{St}$

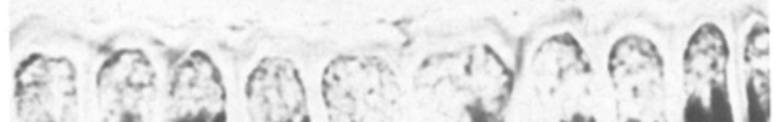

B. istion.

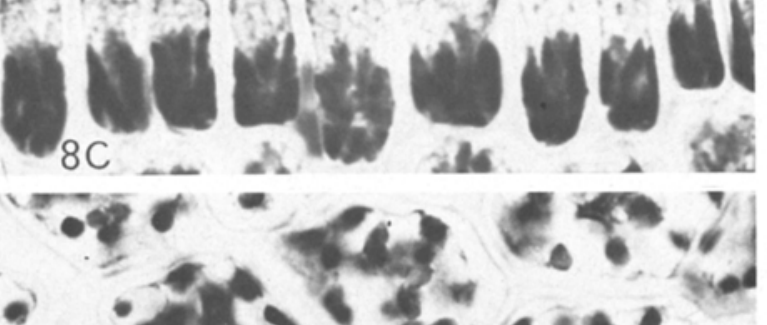

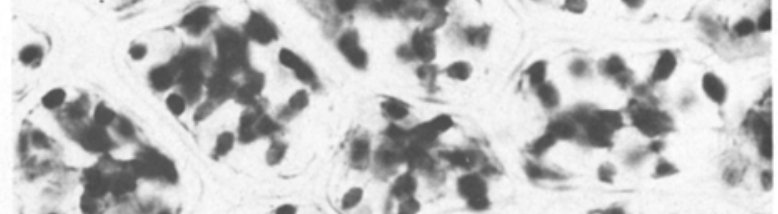

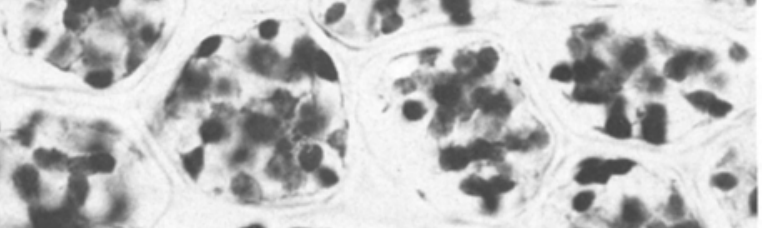

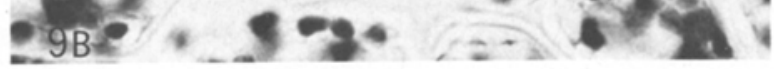
- ang ins:

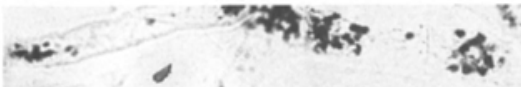

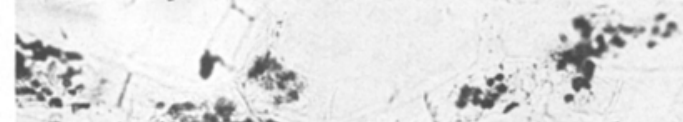
a. 
Thalli, die in Seewasser mit Schwachlicht vorbelichtet waren und während der "halbtrokkenen" bzw. "trockenen" Exposition verdunkelt wurden. Wurden jedoch nach Vorbelichtung mit 20000 Lux in Seewasser die Thalli während der "halbtrockenen" bzw. "trockenen“ Exposition verdunkelt, so waren stärkere Zusammenballungen zu beobachten.

\section{Untersuchungen an Standortproben}

Zum Vergleich mit den Ergebnissen der Untersuchungen im Laboratorium wurden im Helgoländer Nordost-Watt bei Niedrigwasser "halbtrockene" und "trockene" Thalli gesammelt und an Ort und Stelle fixiert. Thalli, die einer nassen Unterlage aufgelegen hatten, also "halbtrocken" waren, zeigten die typische Starklichtanordnung, wenn sie der Sonne direkt exponiert waren. Ein "halbtrockener" Schwachlichtthallus (gemessene Belichtungsbedingungen $800 \mathrm{Lux}$ ) wies eine teilweise Verlagerung der Plastiden in das Innere der Zellen auf. Die untersuchten frei hängenden und infolgedessen stark ausgetrockneten Thalli waren, unabhängig davon, ob sie Starklicht- (25000 Lux) oder Schwachlichtbedingungen' (800 Lux) ausgesetzt waren, häufig stark überwachsen und mehr oder weniger geschädigt. Die Dunkelproben, die bei schwacher Dämmerung entnommen wurden, waren nur leicht getrocknet. Die Chromatophoren der Rindenzellen lagen hier in mehr oder weniger unregelmäßiger Verteilung der Zellwand an. Nur bei den Zellen der inneren Rinde fanden sie sich auch im Zentrum. Im großen und ganzen stimmen also die Beobachtungen an Standortproben mit den Ergebnissen der Laborversuche überein.

\section{Bestrahlungsversuche in hypertonischem Seewasser}

Um Aufschluß darüber zu erhalten, inwieweit die bei "trockenen" und "halbtrockenen" Thalli beobachteten Chromatophorenanordnungen eine Folge der beim Trocknen zunehmenden Salzkonzentration im Thallus sind, wurden Fucus-Thalli in eingeengtem Seewasser (doppelte, drei- und vierfache Konzentration) mit Schwachlicht (1000 Lux) bestrahlt. Infolge der hohen Salzkonzentration verlieren die Thalli ihre Turgeszenz, doch treten keine Plasmolysen auf. Die Plastiden der Rinden- und Epidermiszellen liegen meist verteilt im plasmatischen. Wandbelag und in den Plasmasträngen, die den Zellinnenraum durchziehen (Abb. 7 A). Die Reaktionsstärke ist nicht abhängig vom Hypertoniegrad (vgl. Abb. 7 A, B). Werden die Thalli nach eintägigem Aufenthalt in hypertonischem Seewasser in normales Seewasser überführt, so werden die Pflanzen rasch wieder turgeszent, und die Zellen zeigen bei mikroskopischer Beobachtung keinerlei Schädigungen (vgl. Biebl, 1938). Auch hier wird aber die Plastidenverlagerung nur langsam (innerhalb von 2 Tagen) wieder völlig rückgängig gemacht. Die Plastidenanordnung der Thalli in hypertonischem Seewas-

Abb. 8: Querschnitte durch Thalli, die nach 2tägiger submerser Dunkelvorbehandlung 23 Std. im Dunkeln getrocknet wurden. (A) Epidermis, Rinde und Mark (500:1); (B) Epidermis und Rinde (1000:1); (C) Epidermis (1000:1)

Abb. 9: Flächenschnitte durch Thalli, die wie in Abb. 8 behandelt wurden. (A) Epidermis $(1200: 1)$; (B) äußere Rinde $(800: 1)$; (C) innere Rinde (450:1); (D) Mark (400:1) 
ser entspricht also der bei "trockenen" Thalli beobachteten. Diese Versuche zeigen, daß die bei "halbtrockenen" und "trockenen" Thalli beobachteten Plastidenverlagerungen zum mindesten teilweise auf die beim Trocknen innerhalb des Thallus zunehmende Salzkonzentration zurückzuführen sind und nicht eigentlich eine Reaktion auf die Bestrahlungsverhältnisse darstellen.

\section{DISKUSSION}

Nach den Untersuchungen von Nultsch \& Pfau (1978) kommt das bei Süßwasseralgen, Moosen und höheren Pflanzen weit verbreitete Phänomen der lichtinduzierten Plastidenverlagerungen auch bei zahlreichen Braunalgen vor. Diese Plastidenverlagerungen sind von Absorptionsänderungen begleitet, die allerdings nur wenige Prozent der Gesamtabsorption ausmachen. Nach Ansicht vieler Autoren ermöglichen die Plastidenverlagerungen den Pflanzen, sich an die Lichtbedingungen zu adaptieren: In der Schwachlichtstellung wird die maximal mögliche Absorption erreicht und somit die einfallende Strahlungsenergie optimal photosynthetisch genutzt, während in der Starklichtstellung der Prozentsatz der absorbierten Quanten vermindert ist, was die Pflanzen u. a. vor Strahlungssschäden schützen soll.

Tatsächlich haben Zurzycki $(1955,1975)$ und Lechowski (1974) bei Moosen und höheren Pflanzen gefunden, daß die Photosyntheserate in der Starklichtstellung im Vergleich zur Schwachlichtstellung reduziert war, bei Ajuga reptans z. B. um etwa 50\%, obwohl die durch die Verlagerung hervorgerufene Absorptionsdifferenz nur $6 \%$ betrug.

Nach Rüffer et al. (1977) wird auch bei Fucus vesiculosus die Thallusabsorption mit zunehmender Starklichtlage geringer, wobei allerdings die durch die Chromatophorenverlagerung verursachte Absorptionsdifferenz, bezogen auf die Gesamtabsorption des Thallus, nur etwa $1 \%$ beträgt. Da Nultsch \& Pfau (1979) gezeigt haben, daß die maximale Starklichtlage bei dem bis in die Spritzwasserzone vordringenden $F$. spiralis eher erreicht wird als bei dem im mittleren Eulitoral angesiedelten $F$. vesiculosus, und bei diesem eher als bei dem bis in das untere Eulitoral verbreiteten $F$. serratus, die Dosis-Effekt-Beziehungen bei dieser Gattung also bis zu einem gewissen Grade die Algenzonierung widerspiegeln, ergibt sich die Frage, welche ökologische und physiologische Bedeutung dieses Phänomen für die Algen der Gezeitenzone hat.

Bei der Diskussion dieser Frage ist zu bedenken, daß die Blätter höherer Pflanzen, wenn nicht gerade ein stärkerer Wind weht, in der Regel eine verhältnismäßig konstante Position einnehmen, während die Algen des Eulitorals die meiste Zeit über im Wasser flottieren. Je weiter unten sie im Eulitoral angesiedelt sind, um so seltener fallen sie trocken, und um so kürzer ist auch die Dauer des Trockenfallens. Aber nur während dieses Zeitraumes ist ihre Lage gegenüber der Sonne fixiert, weshalb auch erst mit Beginn des Trockenfallens eine in Beziehung zur Lichtrichtung stehende Chromatophorenverlagerung einsetzen kann. Da nach den Untersuchungen von Rüffer et al. (1977) die Starklichtbewegung bei Fucus etwa 3 bis 4 Std. in Anspruch nimmt, dürfte allerdings für die Pflanzen des mittleren und unteren Eulitorals die Dauer der emersen Phase nur selten zum Erreichen einer kompletten Starklichtstellung ausreichen. Schon unter Beachtung dieser Gesichtspunkte muß man sich die Frage stellen, ob die lichtinduzierten Chromatophorenverlagerungen für die Meeresal- 
gen die gleiche Bedeutung haben können wie für die höheren Pflanzen.

Die vorliegenden Untersuchungen haben gezeigt, daß die Chromatophoren in den Zellen emerser Thalli, und zwar insbesondere nach "trockener" Exposition, keineswegs immer die für die submersen Thalli als typisch erkannten Positionen einnehmen. Besonders die unter Schwachlichtbedingungen zu beobachtende Chromatophorenanordnung weicht von der bei submersen Thalli festgestellten insofern ab, als sich die Chromatophoren stets über die Oberfläche und das Zellinnere mehr oder weniger gleichmäßig verteilen. Diese Anordnung ist innerhalb gewisser Grenzen unabhängig von der Intensität des einfallenden Lichtes und scheint, wie die Untersuchungen mit hypertonischem Seewasser ergeben haben, eher eine Folge des beim Trocknen zwangsläufig erfolgenden Ansteigens der Salzkonzentration im Thallus zu sein. Die durch diese Lageveränderung der Chromatophoren verursachten Transmissionsänderungen konnten zwar nicht gemessen werden, dürften aber insgesamt unerheblich sein.

Diese Beobachtungen sprechen nicht dafür, daß die bei submersen Fucus-Thalli zu beobachtenden lichtinduzierten Chromatophorenverlagerungen eine wesentliche Rolle bei der Regulation der Photosyntheseaktivität emerser Thalli spielen. Die Frage, welche ökologische und physiologische Bedeutung das Phänomen der lichtinduzierten Chromatophorenverlagerung für diese Algen überhaupt hat, bedarf daher noch weiterer Untersuchungen.

Danksagungen. Ein Teil dieser Versuche wurde im photobiologischen Laboratorium der Biologischen Anstalt Helgoland (Meeresstation Helgoland) durchgeführt. Für die Uberlassung des Labors sowie für zahlreiche Hilfeleistungen von seiten der Mitarbeiter der Biologischen Anstalt sagen wir Herrn Prof. Dr. O. Kinne unseren Dank. Der Deutschen Forschungsgemeinschaft danken wir für die Unterstützung der Arbeit.

\section{ZITIERTE LITERATUR}

Biebl, R., 1938. Trockenresistenz und osmotische Empfindlichkeit der Meeresalgen verschieden tiefer Standorte. Jb. wiss. Bot. 86, 350-386.

Kremer, B. P. \& Schmitz, K., 1973. $\mathrm{CO}_{2}$-Fixierung und Stofftransport in benthischen marinen Algen. IV. Zur ${ }^{14} \mathrm{C}$-Assimilation einiger litoraler Braunalgen im submersen und emersen Zustand. $Z$. Pflanzenphysiol. 68, 357-363.

Lechowski, Z., 1974. Chloroplast arrangement as a factor of photosynthesis in multilayered leaves. Acta Soc. Bot. Pol. 43, 531-540.

Montfort, E., 1937. Die Trockenresistenz der Gezeitenpflanzen und die Frage der Ubereinstimmung von Standort und Vegetation. Ber. dt. bot. Ges. 55, 85-95.

Nultsch, W. \& Pfau, J., 1979. Occurrence and biological role of light induced chromatophore displacements in seaweeds. Mar. Biol. 51, 77-82.

Pfau, J., Throm, G. \& Nultsch, W., 1974. Recording microphotometer for determination of light induced chromatophore movements in brown algae. Z. Pflanzenphysiol. 71, 242-260.

Ried, A., 1969. Physiologische Aspekte der Vertikalzonierung von Algen des marinen Litorals. Ber. dt. bot. Ges. 82, 127-141.

Rüffer, U., Nultsch, W. \& Pfau, J., 1977. Untersuchungen zur lichtinduzierten Chromatophorenverlagerung bei Fucus vesiculosus. Helgoländer wiss. Meeresunters. 31, 333-346.

Schramm, W., 1968. Okologisch-physiologische Untersuchungen zur Austrocknungs- und Temperaturresistenz an Fucus vesiculosus L. der westlichen Ostsee. Irit. Revue ges. Hydrobiol. 53, 469-510. 
Stocker, O. \& Holdheide, W., 1938. Die Assimilation Helgoländer Gezeitenalgen während der Ebbezeit. Z. Bot. 32, 1-59.

Zurzycki, J., 1955. Chloroplast arrangements as a factor of photosynthesis. Acta Soc. Bot. Pol. 24, $27-63$.

- 1975. Adjustment processes of the photosynthesis apparatus to light conditions, their mechanism and biological significance. Pol. ecol. Stud. 1, 41-49. 